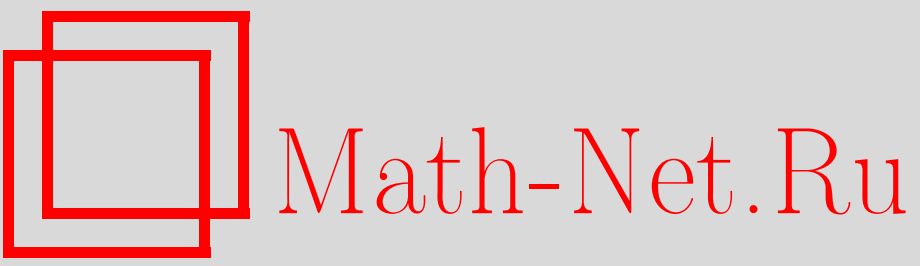

Л. В. Стенюхин, Анализ существования особых решений задачи капиллярности, Итоги науки и техн. Сер. Соврем. мат. и ее прил. Темат. обз., 2019, том $172,113-118$

DOI: https://doi.org/10.36535/0233-6723-2019-172-113-118

Использование Общероссийского математического портала Math-Net.Ru подразумевает, что вы прочитали и согласны с пользовательским соглашением

http://www.mathnet.ru/rus/agreement

Параметры загрузки:

IP: 18.234 .156 .22

26 апреля 2023 г., 13:20:41

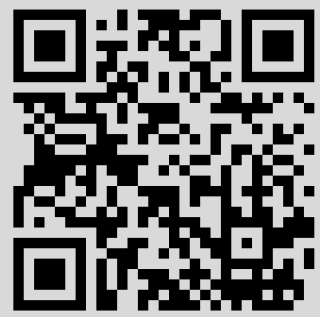




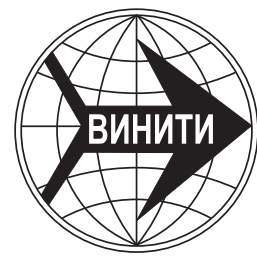

ИТОГИ НАУКИ И ТЕХНИКИ.

Современная математика и ее приложения.

Тематические обзоры.

Том 172 (2019). С. 113-118

DOI: 10.36535/0233-6723-2019-172-113-118

УДК 517.95

\title{
АНАЛИЗ СУЩЕСТВОВАНИЯ ОСОБЫХ РЕШЕНИЙ ЗАДАЧИ КАПИЛЛЯРНОСТИ
}

\author{
(с) 2019 г. Л. В. СТЕНЮХИН
}

\begin{abstract}
АннотАция. Исследуется существование решений капиллярного уравнения при воздействии внешнего потенциала, приводящего к перестройкам поверхности.
\end{abstract}

Ключевые слова: задача капиллярности, число Бонда, бифуркация, особое решение.

\section{ANALYSIS OF THE EXISTENCE OF SPECIAL SOLUTIONS TO THE CAPILLARITY PROBLEM}

\author{
(c) 2019 L. V. STENYUKHIN
}

\begin{abstract}
This paper is devoted to the study of the existence of solutions of the capillary equation under the influence of an external potential leading to surface surgery.
\end{abstract}

Keywords and phrases: capillarity problem, Bond number, bifurcation, special solution.

AMS Subject Classification: 35R01

1. Постановка задачи и комментарии к предмету исследования. Равновесные устойчивые и неустойчивые формы капель и капиллярных трубок исследовались в $[2,6,7,9,10]$. Функции, задающие эти формы, являются решениями уравнения капиллярности и обычно ищутся итерационными методами в виде рядов. Однако если размеры форм достаточно большие либо фирмы подвергаются воздействию потенциала, то нарушается сходимость итераций или полученные решения начинают противоречить физическим экспериментам (см. [9]). Разрешимость уравнения капиллярности в классах обобщенных функций была доказана Н. Н. Уральцевой (см. [8]).

Капиллярное уравнение возникает из вариационного принципа минимизации энергетического функционала, состоящего из энергии поверхностного натяжения, потенциальной энергии силы тяжести и энергии объемных связей. Энергия поверхностного натяжения определяется функционалом площади, исследованию которого посвящены работы $[1,2,7]$. Экстремали функционала площади определяют минимизацию энергии поверхностного натяжения.

Настоящая работа посвящена исследованию существования решений капиллярного уравнения при воздействии внешнего потенциала, приводящего к перестройкам поверхности. Вариационный принцип в банаховых и гильбертовых пространствах и операторный подход (см. [3,4]) позволяют находить такие решения, критические значения параметров и соответствующие формы капель и трубок.

Рассмотрим связную каплю жидкости заданного объема $V$, лежащую на горизонтальной плоскости П в поле силы тяжести, направленном вертикально вниз. Предположим, что материал жидкости однороден, так что угол контакта жидкости с плоскостью $\gamma$ постоянен, $0 \leqslant \gamma \leqslant \pi$. Как показано в [10], при этих условиях равновесная поверхность будет поверхностью вращения с осью перпендикулярной плоскости П. 
Задача о малой капле, лежащей на поверхности, является следствием минимизации следующих энергий:

(1) энергии поверхностного натяжения;

(2) потенциальной энергии силы тяжести;

(3) энергии объемных связей (постоянства объема $V$ ).

Полная энергия определяется функционалом

$$
E(u)=\int_{\Omega} \sqrt{E G-F^{2}} d x+\frac{1}{\sigma} \int_{\Omega} \Upsilon \rho u d x+\lambda \int_{\Omega} u d x,
$$

где $E, F, G$ - коэффициенты первой квадратичной формы поверхности, $\sigma$ - поверхностное натяжение, $\Upsilon$ - потенциальная энергия на единицу массы, $\rho$ - плотность, $\lambda$-множитель Лагранжа, отвечающий за объем.

В точках верхней части свободной поверхности $P$ капли высота $u(x, y)$ поверхности $P$ над П удовлетворяет уравнению

$$
\operatorname{div} T u=\chi u+\lambda
$$

где

$$
T u=\frac{1}{\sqrt{1+|\nabla u|^{2}}} \nabla u, \quad \nabla u=\left(u_{x}, u_{y}\right),
$$

$\chi=\rho g / \sigma$. Для нижней части свободной поверхности знак $\operatorname{div} T u$ надо изменить на противоположный. Граничное условие задачи с постоянным углом контакта $\gamma$ имеет вид

$$
\boldsymbol{n} T u=\cos \gamma,
$$

$\boldsymbol{n}$ - единичная нормаль.

Замена $u=-v-(1 / \chi) \lambda$ переводит уравнение (1) в уравнение

$$
\operatorname{div} T v=\chi v,
$$

которое является уравнением свободной поверхности в капиллярной трубке. Каждому уравнению (1) с высотой капли в центре $u_{0}$ соответствует решение уравнения (3) с высотой подъема жидкости в центре $v_{0}=-\left(u_{0}+\lambda / \chi\right)$. В [9] показано, что симметричные решения уравнения (3) однозначно определяются высотой подъема жидкости в центре. Поэтому каждой симметрично лежащей капле отвечает единственная капиллярная поверхность, которая (локально) геометрически конгруэнтна границе капли. Обратно, каждой симметричной капиллярной поверхности соответствует лежащая капля, определенная с точностью до аддитивной константы.

Множество всех симметричных капиллярных поверхностей является однопараметрическим семейством с параметром центральной высоты $u_{0}$ поверхности. Из принципа соответствия вытекает, что множество всех симметрично лежащих капель может быть описано с помощью однопараметрического семейства кривых.

С учетом вышеизложенного, уравнение (1) можно записать в безразмерной форме

$$
\operatorname{div} T u=B u,
$$

$B$ - число Бонда, характеризующее размер конфигурации: $B=\rho g a^{2} / \sigma$. Граничное условие (2) сохраняется.

2. Частные случаи существования особых решений. В [9] показано, что для симметрично лежащей капли, $0<\gamma<\pi / 2$ и малого числа Бонда $B$ уравнение (4) можно записать в виде

$$
\operatorname{div} \frac{\nabla u}{\sqrt{1+|\nabla u|^{2}}}=B u-2 \sin \gamma_{0}
$$


Решение ищется на круге $\Omega, u=0$ на $\partial \Omega$, и капля постоянного объема $V_{0}$. Если капля подвергается воздействию внешнего потенциала $\varphi$, то

$$
\begin{gathered}
V_{0}=\frac{\pi\left(2+\cos \gamma_{0}\right)\left(1-\cos \gamma_{0}\right)^{2}}{3 \sin ^{3} \gamma_{0}}, \\
\cos \gamma_{0}=\cos \gamma+\frac{\varphi}{\sigma}
\end{gathered}
$$

Потенциал $\varphi$ может быть, например, температурой вещества капли или давлением, воздействующим изнутри капли, либо температурой и давлением одновременно. Действие потенциала приводит к изменению формы капли, в частности, к изменению угла контакта с плоскостью П (см. (7)). При этом $\gamma_{0}$ является единственным решением уравнения (6). Дальнейшее воздействие потенциала $\varphi$ приведет к изменению числа Бонда $B$ и к перестройке (бифуркации) самой капли.

Для описания дальнейших состояний капли положим $B=0$ в уравнении (5).

Теорема 1. При сделанных выше предположениях и $B=0$ существует точное решение уравнения (5):

$$
u_{0}=\frac{-\cos \gamma_{0}+\sqrt{1-r^{2} \sin ^{2} \gamma_{0}}}{\sin \gamma_{0}} .
$$

Решение (8) проверяется непосредственной проверкой. Это решение послужит отправной точкой для отыскания дальнейших состояний капли, подверженной воздействию потенциала $\varphi$.

Для ненулевых чисел $B$ положим, что решение уравнения (5) примет вид

$$
u=\frac{-\cos \gamma_{0}+\sqrt{1-\left(r-r_{0}\right)^{2} \sin ^{2} \gamma_{0}}}{\sin \gamma_{0}}
$$

$r_{0}-$ радиус кольца по центру, $\left|r-r_{0}\right| \leqslant 1$.

Непосредственным вычислением получим, что функция (9) является точным решением уравнения

с нулевым граничным условием.

$$
\operatorname{div} \frac{\nabla u}{\sqrt{1+|\nabla u|^{2}}}=\frac{r_{0} \sin \gamma_{0}}{r}-2 \sin \gamma_{0}
$$

Найдем условия, при которых задача (10) эквивалентна задаче (5). Для этого положим

т.e.

$$
B u=\frac{r_{0} \sin \gamma_{0}}{r}
$$

$$
\frac{-\cos \gamma_{0}+\sqrt{1-\left(r-r_{0}\right)^{2} \sin ^{2} \gamma_{0}}}{\sin \gamma_{0}}=\frac{r_{0} \sin \gamma_{0}}{B r}
$$

Последнее уравнение преобразуется к виду

$$
\left(1+\frac{\sin ^{2} \gamma_{0}}{B^{2} r^{2}}\right) r_{0}^{2}-2\left(r-\frac{\cos \gamma_{0}}{B r}\right) r_{0}+r^{2}-1=0 .
$$

Разрешим уравнение (11) относительно $r_{0}$, как гарант кольцеобразного состояния:

$$
D_{1}=\left(\frac{\cos \gamma_{0}}{B}-1\right)^{2}+\frac{1-r^{2}}{B^{2} r^{2}}
$$

В решении (8) безразмерного уравнения (5) полярная координата $r$ меняется в пределах $0 \leqslant r \leqslant 1$; значит, с увеличением потенциала $\varphi$ у деформированной капли значение $r=1$ тоже существует. Равенство нулю выражения (12) для $r=1$ порождает условие

$$
B=\cos \gamma_{0} .
$$

Таким образом, получается следующая теорема.

Теорема 2. Если выполнено условие (13), то существует точное аналитическое решение задачи (5) типа (9) с граничным условием (2). 
3. Редукция задачи из функционала площади. Рассмотрим основной энергетический функционал задачи

$$
E(u)=\int_{\Omega} \sqrt{E G-F^{2}} d x+\frac{1}{\sigma} \int_{\Omega} \Upsilon \rho u d x+\lambda \int_{\Omega} u d x .
$$

Его первое слагаемое является функционалом площади. Пусть $u_{0}$ - экстремаль функционала (14). Близкие к $u_{0}$ поверхности будем задавать в системе координат нормального расслоения к $u_{0}$. Это приведет к одному скалярному уравнению для близкой поверхности:

$$
\left(\frac{\delta S}{\delta u}\left(u_{0}+\eta \bar{n}\right), \bar{n}\right)=0
$$

или

$$
\frac{\delta S}{\delta \eta}(\eta)=0
$$

где $\delta S / \delta u$ - функциональная производная функционала площади, $\bar{n}-$ нормаль к поверхности $u_{0}$. Из уравнения (15) определяется нормальная координата $\eta=\eta(x, y)$.

Теорема 3. Функиионал площади близких $к u_{0}$ поверхностей $S(\eta)$ и его оператор Эйлера $\delta S / \delta \eta(\eta)$ имеет следующую аналитическую структуру:

$$
\begin{gathered}
S(u)=\int_{\Omega} \sqrt{E G-F^{2}} d x d y \\
\frac{\delta S}{\delta u}(\eta)=E^{3}\left(E G-F^{2}\right)^{-3 / 2}\left(A \eta_{x x}-2 B \eta_{x y}+C \eta_{y y}+G\right) .
\end{gathered}
$$

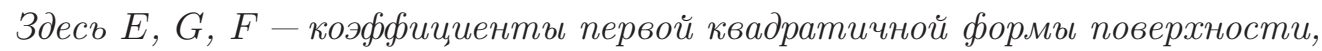

$$
\begin{gathered}
A=\sum_{p=1}^{6} a_{i j k} \eta_{x}^{i} \eta_{x}^{j} \eta^{k}+1, \quad B=\sum_{p=1}^{6} b_{i j k} \eta_{x}^{i} \eta_{y}^{j} \eta^{k}, \quad C=\sum_{p=1}^{6} c_{i j k} \eta_{y}^{i} \eta_{y}^{j} \eta^{k}+1 \\
G=\sum_{p=2}^{7} g_{i j k} \eta_{x}^{i} \eta_{y}^{j} \eta^{k}+g \eta
\end{gathered}
$$

где $i, j, k$ - целье неотрицательные числа, $p=i+j+k$. Все коэффициенты $a_{i j k}, b_{i j k}, c_{i j k}, g_{i j k}$, $g$ являются аналитическими функииями и находятся по формулам, подобным следующей:

$$
g=\left(\bar{n}, \bar{n}_{x x}+\bar{n}_{y y}\right)+\frac{4}{E}\left[\left(\bar{n}, u_{x x}\right)^{2}+\left(\bar{n}, u_{y y}\right)^{2}\right] .
$$

Линейная часть оператора $A \eta_{x x}-2 B \eta_{x y}+C \eta_{y y}+G$ равна $\Delta \eta+g \eta$, где $\Delta$ - лапласиан. Первая вариация функционала энергии (14) равна

$$
\frac{\delta E}{\delta \eta}(\eta)=\frac{\delta S}{\delta \eta}(\eta)+\left(\frac{\Upsilon \rho}{\sigma}+\lambda\right) \eta=E^{3}\left(E G-F^{2}\right)^{-3 / 2}\left(A \eta_{x x}-2 B \eta_{x y}+C \eta_{y y}+G\right)+\left(\frac{\Upsilon \rho}{\sigma}+\lambda\right) \eta .
$$

Линейная часть первой вариации равна

$$
L \eta=E^{3}\left(E G-F^{2}\right)^{-3 / 2}(\Delta \eta+g \eta)+\left(\frac{\Upsilon \rho}{\sigma}+\lambda\right) \eta
$$

где величина $g$ определена равенством (16). Выражение $\Upsilon \rho / \sigma$ определяет число Бонда, $B=\Upsilon \rho / \sigma$. Поэтому линеаризованная задача имеет вид

$$
\Delta \eta+\left(g+E^{-3}\left(E G-F^{2}\right)^{3 / 2}(B+\lambda)\right) \eta-0,\left.\quad \eta\right|_{\partial \Omega}=0 .
$$

Линейный оператор $(17)$ действует из $W_{2}^{2}(\Omega)$ в $L_{2}(\Omega)$ и самосопряжен в $\stackrel{\circ}{W}_{2}^{2}(\Omega)$ относительно скалярного произведения в $L_{2}(\Omega)$. 
Приведем пример из п. 2. Функция (8) является решением задачи (5)-(7) при нулевых чисел Бонда. Задача (17) в данном случае имеет вид

$$
\Delta \eta=\left(\frac{\sqrt{\left(1-\left(x^{2}+y^{2}\right) \sin ^{2} \gamma_{0}\right)^{3}}}{\left(1-y^{2} \sin ^{2} \gamma_{0}\right)^{3}} B-g\right) \eta+\left(\frac{\sqrt{\left(1-\left(x^{2}+y^{2}\right) \sin ^{2} \gamma_{0}\right)^{3}}}{\left(1-y^{2} \sin ^{2} \gamma_{0}\right)^{3}}\right) \lambda \eta,
$$

где

$$
\begin{array}{r}
g=\frac{\sin ^{4} \gamma_{0}\left(3 x^{2}+3 y^{2}-3 x^{2} y^{2} \sin ^{2} \gamma_{0}+3 x^{3} y \sin ^{2} \gamma_{0}+y(x+y)\left(1-\left(x^{2}+y^{2}\right) \sin ^{2} \gamma_{0}\right)\right)}{\left(1-\left(x^{2}+y^{2}\right) \sin ^{2} \gamma_{0}\right)^{5}}+ \\
+\frac{4 \sin ^{2} \gamma_{0}\left(1+\left(1-\left(x^{2}+y^{2}\right) \sin ^{2} \gamma_{0}\right)^{2}\right)}{\left(1-y^{2} \sin ^{2} \gamma_{0}\right)\left(1-\left(x^{2}+y^{2}\right) \sin ^{2} \gamma_{0}\right)^{2}},
\end{array}
$$

и $\left.\eta\right|_{\partial \Omega}=0$. Здесь $\Omega$-единичный двумерный диск.

На малом диске $\Omega_{\varepsilon}=\left\{x^{2}+y^{2} \leqslant \varepsilon\right\}$ около центра области $\Omega$ имеем $g=8 \sin ^{2} \gamma_{0}+o(x, y)$, и задача (18) соответствует задаче

$$
\Delta \eta=\left(B-8 \sin ^{2} \gamma_{0}+\lambda\right) \eta,\left.\quad \eta\right|_{\partial \Omega_{\varepsilon}}=0 .
$$

В результате получается следующая теорема.

Теорема 4. Если $B-8 \sin ^{2} \gamma_{0}=\mu_{n}, \mu_{n}$ - собственное значение лапласиана $\Delta$ на области $\Omega_{\varepsilon}$, то капиллярная поверхность вблизи начала координат задается функиией

$$
u_{n}=u_{0}+\varepsilon \eta_{n},
$$

где $u_{0}$ - функиия (8), $\eta_{n}$ - собственная функиия, отвечающая собственному значению $\mu_{n}$.

4. Задача капиллярности в конформных координатах. Пусть поверхность капли $u(x, y)=\left(u_{1}(x, y), u_{2}(x, y), u_{3}(x, y)\right)$ задана в конформных координатах, $E=G, F=0$. Тогда функция $u(x, y)$ удовлетворяет условиям $u_{x}^{2}=u_{y}^{2}, u_{x} u_{y}=0$. В этом случае функционал энергии имеет вид

$$
E(u)=\int_{\Omega} \frac{E+G}{2} d x+\int_{\Omega} B u d x+\lambda \int_{\Omega} u d x .
$$

Первая вариация функционала равна

Получаем задачу

$$
\frac{\delta E}{\delta u}(\eta)=\Delta \eta+(B+\lambda) \eta
$$

$$
\Delta \eta+(B+\lambda) \eta-0,\left.\quad \eta\right|_{\partial \Omega}=0
$$

Теорема 5. Собственнъе значения оператора $\Delta+B+\lambda$ задачи (19) равнъ $\tilde{\lambda}_{n}=\lambda_{n}+B+\lambda$, где $\lambda_{n}$-собственные значения оператора $\Delta$ с нулевым граничным условием.

Таким образом, переход к конформным координатам упрощает нахождение спектра и собственных функций оператора капиллярности. Поверхность (8) стереографически спроектируем на $\mathbb{R}^{2}$. Пусть $\tilde{u}_{0}(r)$ - соответствующая функция после проектирования,

$$
\tilde{u}_{0}(r)=\frac{u_{0}(0) r}{u_{0}(0)-u_{0}(r)}, \quad u_{0}(r)=\frac{-\cos \gamma_{0}+\sqrt{1-r^{2} \sin ^{2} \gamma_{0}}}{\sin \gamma_{0}},
$$

$\tilde{\eta}_{n}$ - собственные функции для $\tilde{\lambda}_{n}$ оператора задачи $(19)$. Тогда возмущения вида $\tilde{u}_{n}(r)=\tilde{u}_{0}(r)+$ $\varepsilon \tilde{\eta}_{n}(r)$ порождают совокупность капиллярных поверхностей

$$
u_{n}(r)=u_{0}(0) \frac{\tilde{u}_{n}(r)-r}{\tilde{u}_{n}(r)} .
$$




\section{СПИСОК ЛИТЕРАТУРЫ}

1. Борисович А. Ю. Оператор Плато и бифуркации двумерных минимальных поверхностей// в кн.: Глобальный анализ и математическая физика. - Воронеж, 1987. - С. 142-155.

2. Дао Чонг Тхи, Фоменко А. Т. Минимальные поверхности и проблема Плато. - М.: Наука, 1987.

3. Даринский Б. М., Сапронов Ю. И., Царев С. Л. Бифуркации экстремалей фредгольмовых функционалов// Совр. мат. Фундам. направл. - 2004. - 12. - С. 3-140.

4. Ниренберг Л. Лекции по нелинейному функциональному анализу. - М.: Мир, 1987.

5. Стенюхин Л. В. О минимальных поверхностях с ограничениями типа неравенств// Изв. вузов. Мат. - 2012. - 11. - C. 51-59.

6. Стенюхин Л. В. Об особых решениях задачи капиллярности с круговой симметрией// Вестн. Воронеж. гос. ун-та. Сер. Физ. Мат. - 2013. - 2. - С. 242-245.

7. Стенюхин Л. В. Бифуркационный анализ задачи капиллярности с круговой симметрией// Вестн. Южно-Урал. гос. ун-та. Сер. Мат. модел. програм. - 2014. - 7, № 2. - С. 77-83.

8. Уральцева Н. Н. Решение задачи капиллярности// Вестн. ЛГУ - 1973. - 19. - С. 54-64.

9. Финн Р. Равновесные капиллярные поверхности. Математическая теория. - М.: Мир, 1989.

10. Wente H. C. The symmetry of sessile and pendent drops// Pac. J. Math. — 1980. — 88, № 2 . P. 387-397.

Стенюхин Леонид Витальевич

Воронежский государственный университет

E-mail: stenyuhin@mail.ru 